\title{
DIVERSIDADE E SIMILARIDADE DE FRAGMENTOS FLORESTAIS NATIVOS SITUADOS NA REGIÃO NORDESTE DE MINAS GERAIS
}

\author{
Christianne Riquetti Corsini ${ }^{1}$, José Roberto Soares Scolforo ${ }^{2}$, Antônio Donizette de Oliveira ${ }^{2 *}$, José Márcio de Mello ${ }^{2}$, \\ Evandro Luiz Mendonça Machado ${ }^{3}$
}

*Autor para correspondência: donizete@dcf.ufla.br

RESUMO: Nesse estudo foram amostrados 26 fragmentos de floresta nativa distribuídos em quatro Bacias hidrográficas da região nordeste de Minas Gerais, com o objetivo de analisar a similaridade florística existente entre 26 fragmentos florestais nativos inseridos em quatro bacias hidrográficas do nordeste do estado de Minas Gerais e a diversidade e equabilidade das fisionomias estudadas e dos grupos de fragmentos formados. Utilizou-se amostragem sistemática com unidades amostrais de $1000 \mathrm{~m}^{2}$ cada, onde a área amostral variou de 1 a 6 ha, conforme a área do fragmento. Foram mensurados a circunferência a 1,30 m do solo (CAP) e a altura total e coletado material botânico de todos os indivíduos com CAP maior ou igual a 15,7cm. Formaram-se seis grupos florísticos de acordo com o coeficiente de similaridade de Sorensen, sendo que em quatro deles houve associação de mais de uma fisionomia, evidenciando regiões de transição dentro da área. O índice de diversidade de Shannon, variou de 2,236 na Floresta Estacional Decidual a 4,523 na Floresta Estacional Semidecidual. Os valores máximo e mínimo da equabilidade medida pelo índice de Pielou foram de 0,850 e 0,616, respectivamente. O grupo florístico 2 (Floresta Estacional Semidecidual e Cerrado sensu stricto) apresentou o maior valor médio de diversidade $(3,585)$ e equabilidade $(0,750)$, e o grupo 1 (Floresta Estacional Decidual) apresentou os menores valores $\left(H^{\prime}\right.$ : 2,426 e J': 0,687).

Palavras-chave: equabilidade, fisionomias florestais, grupos florísticos

\section{DIVERSITY AND SIMILARITY OF NATIVE FOREST FRAGMENTS LOCATED IN THE NORTHEAST REGION OF MINAS GERAIS}

\begin{abstract}
In this study 26 distributed fragments of native forest in four located hydrographical Basins in the northeast region of Minas Gerais had been showed, with the purpose of analyzing the floristic similarity exists between 26 forest fragments native inserted in four basins in the northeast of the state of Minas Gerais and the diversity and evenness of physiognomies studied and groups of fragments formed. Systematic sampling with units was used shows of $1000 \mathrm{~m}^{2}$ each, where the sample area varied of 1 the 6 has, as the area I break up of it. We measured the circumference at $1.30 \mathrm{~m}(\mathrm{CAP})$ and the total height and collected botanical material of all individuals with CAP greater or equal to $15.7 \mathrm{~cm}$. Six groups were formed according to the floristic similarity coefficient Sorensen, with four groups there was an association of more than a physiognomy, showing regions of transition within the area. The Shannon diversity index, ranged from 2.236 in deciduous forest to 4.523 in Semideciduous Forest. The maximum and minimum values of evenness index Pielou were 0.850 and 0.616 , respectively. The floristic group 2 (Semideciduous Forest and Cerrado sensu stricto) had the highest average value of diversity (3.585) and evenness (0.750), and group 1 (Deciduous Forest) had the lowest values ( $H^{\prime}: 2.426$ and $\left.J^{\prime}: 0.687\right)$.
\end{abstract}

Key-words: wealth, equability, hydrographical Basins, forest formations.

\section{INTRODUÇÃO}

O processo de fragmentação afeta a organização das comunidades naturais, especialmente porque reduz a área de vida das espécies e altera as condições climáticas locais (BIERREGAARD; DALE, 1996). As pressões antrópicas sobre a vegetação nativa colocaram em risco a alta riqueza de espécies em Minas Gerais, que é resultado da grande diversidade de fisionomias vegetais que compõem os Biomas presentes no Estado: Mata Atlântica, Cerrado e Caatinga (COSTA et al., 1998).
De 1987 a 1992, a utilização dos recursos florestais foi da ordem de 2.848.627,61 ha, sendo $60,38 \%$ representados por cobertura nativa e apenas $39,62 \%$ por florestas plantadas (CARVALHO, 1996). Segundo Scolforo e Carvalho (2006), no período entre 2003 e 2005 houve redução de 152.635 hectares na área ocupada com flora nativa no Estado de Minas Gerais.

Segundo Carvalho et al. (2005) é de extrema importância o estudo da diversidade biológica contida nos atuais fragmentos para avaliação dos potenciais de perdas

${ }^{1}$ Belo Horizonte, Minas Gerais, Brasil

${ }^{2}$ Universidade Federal de Lavras - Lavras, Minas Gerais, Brasil

${ }^{3}$ Universidade Federal dos Vales do Jequitinhonha e Mucuri - Diamantina, Minas Gerais, Brasil

Cerne, Lavras, v. 20, n. 1, p. 1-10, jan./mar. 2014 
e conservação dos recursos naturais a longo prazo. Esses remanescentes têm um papel importante como corredores ecológicos, capazes de proporcionar o fluxo gênico entre populações isoladas, um fator tido como crítico para a conservação de muitas espécies (KAGEYAMA; GANDARA, 2000; SILVA; TABARELLI, 2000).

Dessa forma, o conhecimento das relações entre a composição florística e da diversidade dos ecossistemas é de suma importância no conhecimento de padrões florísticos de fragmentos ajudando na descrição e análise da flora das formações vegetais onde estão inseridos e na determinação da similaridade entre áreas.

Os objetivos desse estudo foram analisar a similaridade florística existente entre 26 fragmentos florestais nativos inseridos em quatro bacias hidrográficas do nordeste do estado de Minas Gerais e a diversidade e equabilidade das fisionomias estudadas e dos grupos de fragmentos formados.

\section{MATERIAL E MÉTODOS}

\subsection{Descrição da área de estudo}

A área de estudo compreende as Bacias Hidrográficas dos rios Jequitinhonha, Mucuri, Pardo e São Mateus, localizadas na região nordeste de Minas Gerais e compreende uma área de 9.882 .500 ha. Nessa área foram amostrados 26 fragmentos florestais com o propósito de representar as diferentes fitofisionomias existentes nessas Bacias.

Os fragmentos estão identificados neste trabalho por meio de um código composto da letra inicial da Bacia a qual pertence e um número de identificação. A classificação fisionômica dos fragmentos foi obtida do Mapeamento e Inventário da Flora Nativa e dos Reflorestamentos de Minas Gerais (SCOLFORO; CARVALHO, 2006) e os dados ambientais foram provenientes do Zoneamento Ecológico Econômico do Estado de Minas Gerais (CARVALHO; SCOLFORO, 2007). A localização dos fragmentos, bem como a classificação fisionômica e a caracterização ambiental dos mesmos consta na Tabela 1.

Tabela 1 - Localização dos fragmentos amostrados e a classificação fisionômica dos mesmos.

Table 1 - Location of the sampled fragments and theirs physiognomic classification.

\begin{tabular}{|c|c|c|c|c|c|c|c|c|c|}
\hline Bacia hidrográfica & Município & $\begin{array}{l}\text { Identificação do } \\
\text { Fragmento }\end{array}$ & $\begin{array}{c}\text { Fisionomia } \\
\text { predomi- } \\
\text { nante** }\end{array}$ & Longitude & Latitude & $\begin{array}{l}\text { Altitude } \\
\text { média (m) }\end{array}$ & $\begin{array}{l}\text { Precipitação } \\
\text { média (mmm) }\end{array}$ & $\begin{array}{l}\text { Temperatura } \\
\text { média }\left({ }^{\circ} \mathrm{C}\right)\end{array}$ & $\begin{array}{l}\text { Solo Predom- } \\
\text { inante }\end{array}$ \\
\hline São Mateus & Frei Gaspar & S111 & FES & $40^{\circ} 50^{\prime} 07^{\prime \prime}$ & $17^{\circ} 01^{\prime} 16^{\prime \prime}$ & 338 & 905 & 24,7 & Latossolo \\
\hline Jequitinhonha & Araçuaí & $\mathrm{J} 110$ & CSS & $42^{\circ} 07^{\prime} 45^{\prime \prime}$ & $16^{\circ} 08^{\prime} 33^{\prime \prime}$ & 845 & 913 & 22,7 & Cambissolo \\
\hline Jequitinhonha & Aricanduva & J114 & FED & $42^{\circ} 15^{\prime} 51^{\prime \prime}$ & $16^{\circ} 14^{\prime} 12^{\prime \prime}$ & 467 & 906 & 23,8 & Nitossolo \\
\hline Jequitinhonha & Coronel Murta & J120 & CSS & $43^{\circ} 47^{\prime} 47^{\prime \prime}$ & $17^{\circ} 20^{\prime} 50^{\prime \prime}$ & 876 & 1187 & 20,9 & Latossolo \\
\hline Jequitinhonha & Fruta de Leite & $\mathrm{J} 86$ & CSS & $43^{\circ} 46^{\prime} 27^{\prime \prime}$ & $17^{\circ} 17^{\prime} 56^{\prime \prime}$ & 855 & 1187 & 20,9 & Latossolo \\
\hline Jequitinhonha & Itacambira & J96 & CSS & $42^{\circ} 22^{\prime} 11^{\prime \prime}$ & $17^{\circ} 19^{\prime} 26^{\prime \prime}$ & 1003 & 1035 & 21,8 & Argissolo \\
\hline Jequitinhonha & Itamarandiba & $\mathrm{J} 13$ & CSS & $41^{\circ} 13^{\prime} 32^{\prime \prime}$ & $16^{\circ} 38^{\prime} 23^{\prime \prime}$ & 857 & 932 & 22,2 & Latossolo \\
\hline Jequitinhonha & Jacinto & $\mathrm{J} 135$ & CSS & $42^{\circ} 46^{\prime} 05^{\prime \prime}$ & $17^{\circ} 44^{\prime} 17^{\prime \prime}$ & 944 & 1089 & 20,4 & Latossolo \\
\hline Jequitinhonha & Jenipapo de Minas & $\mathrm{J} 20$ & FED & $42^{\circ} 12^{\prime} 44^{\prime \prime}$ & $17^{\circ} 07^{\prime} 22^{\prime \prime}$ & 615 & 960 & 23,4 & Argissolo \\
\hline Jequitinhonha & Jequitinhonha * & $\mathrm{J} 108$ & FES & $41^{\circ} 26^{\prime} 47^{\prime \prime}$ & $17^{\circ} 44^{\prime} 55^{\prime \prime}$ & 571 & 1042 & 23,1 & Latossolo \\
\hline Jequitinhonha & Jequitinhonha $*$ & J109 & $\mathrm{CC}$ & $42^{\circ} 17^{\prime} 00^{\prime \prime}$ & $15^{\circ} 59^{\prime} 20^{\prime \prime}$ & 890 & 950 & 21,8 & Cambissolo \\
\hline Jequitinhonha & Leme do Prado & $\mathrm{J} 10$ & FES & $40^{\circ} 26^{\prime} 19^{\prime \prime}$ & $17^{\circ} 44^{\prime} 29^{\prime \prime}$ & 220 & 946 & 25,5 & Latossolo \\
\hline Jequitinhonha & Minas Novas & J14 & FED & $42^{\circ} 06 ' 37^{\prime \prime}$ & $16^{\circ} 54^{\prime} 39^{\prime \prime}$ & 354 & 876 & 24,4 & Argissolo \\
\hline Jequitinhonha & Olhos d'água * & J11 & FES & $41^{\circ} 38^{\prime} 50^{\prime \prime}$ & $16^{\circ} 07^{\prime} 41^{\prime \prime}$ & 876 & 891 & 22,3 & Latossolo \\
\hline Jequitinhonha & Olhos d'água * & $\mathrm{J} 12$ & CSS & $42^{\circ} 17^{\prime} 00^{\prime \prime}$ & $16^{\circ} 36^{\prime} 15^{\prime \prime}$ & 777 & 921 & 23,8 & Latossolo \\
\hline Jequitinhonha & Salinas * & J16 & FES & $40^{\circ} 21^{\prime} 33^{\prime \prime}$ & $16^{\circ} 08^{\prime} 20^{\prime \prime}$ & 214 & 839 & 25,6 & Argissolo \\
\hline Jequitinhonha & Salinas * & $\mathrm{J} 21$ & CSS & $42^{\circ} 45^{\prime} 53^{\prime \prime}$ & $17^{\circ} 09^{\prime} 57^{\prime \prime}$ & 898 & 1054 & 21,6 & Cambissolo \\
\hline Jequitinhonha & São G. do Rio Preto & J139 & FES & $42^{\circ} 31^{\prime} 43^{\prime \prime}$ & $17^{\circ} 52^{\prime} 40^{\prime \prime}$ & 873 & 1101 & 20,7 & Latossolo \\
\hline Jequitinhonha & Turmalina & $\mathrm{J} 15$ & CSS & $43^{\circ} 16^{\prime} 10^{\prime \prime}$ & $16^{\circ} 55^{\prime} 00^{\prime \prime}$ & 915 & 1081 & 20,5 & Cambissolo \\
\hline Mucuri & Águas Formosas & M140 & CSS & $42^{\circ} 21^{\prime} 05^{\prime \prime}$ & $15^{\circ} 06^{\prime} 11^{\prime \prime}$ & 985 & 867 & 21,9 & Latossolo \\
\hline Mucuri & Nanuque & M134 & $\mathrm{CC}$ & $42^{\circ} 49^{\prime} 09^{\prime \prime}$ & $17^{\circ} 24^{\prime} 05^{\prime \prime}$ & 823 & 1077 & 21,7 & Cambissolo \\
\hline Mucuri & Teófilo Otoni & M112 & FES & $43^{\circ} 23^{\prime} 51^{\prime \prime}$ & $17^{\circ} 59^{\prime} 35^{\prime \prime}$ & 825 & 1236 & 20,2 & Cambissolo \\
\hline Pardo & Indaiabira & P119 & CSS & $42^{\circ} 09^{\prime} 52^{\prime \prime}$ & $15^{\circ} 28^{\prime} 48^{\prime \prime}$ & 887 & 618 & 21,8 & Latossolo \\
\hline Pardo & Montezuma & P94 & $\mathrm{CC}$ & $42^{\circ} 29^{\prime} 52^{\prime \prime}$ & $15^{\circ} 35^{\prime} 49^{\prime \prime}$ & 963 & 907 & 22,3 & Cambissolo \\
\hline Pardo & Ninheira & P115 & FES & $41^{\circ} 34^{\prime} 49^{\prime \prime}$ & $15^{\circ} 24^{\prime} 08^{\prime \prime}$ & 851 & 860 & 22,1 & Cambissolo \\
\hline Pardo & Rio Pardo de Minas & P95 & FES & $41^{\circ} 04^{\prime} 31^{\prime \prime}$ & $18^{\circ} 04^{\prime} 31^{\prime \prime}$ & 538 & 1051 & 23,2 & Latossolo \\
\hline
\end{tabular}

* Fragmentos situados no mesmo município, mas em propriedades diferentes.

** FES: Floresta Estacional Semidecidual; FED: Floresta Estacional Decidual; CSS: Cerrado sensu stricto; CC: Campo cerrado

Cerne, Lavras, v. 20, n. 1, p. 1-10, jan./mar. 2014 


\subsection{Amostragem e coleta de variáveis dendrométricas}

Utilizou-se o procedimento de amostragem sistemático com parcelas de $1000 \mathrm{~m}^{2}$ (10x100 metros). O número de parcelas lançadas em cada fragmento foi relativo ao seu tamanho, sendo a intensidade amostral de uma parcela a cada 15 ha (Tabela 2). Assim, a distância entre unidades amostrais também variou conforme o tamanho do fragmento. Mediram-se a altura e a circunferência a altura de 1,3 m (CAP) de todas as árvores cuja CAP foi igual ou superior a $15,7 \mathrm{~cm}$.

Coletou-se material botânico de todos os indivíduos e as exsicatas foram encaminhadas ao herbário da Universidade Federal de Lavras para identificação por especialistas, que usaram como referência Angiosperm Phylogeny Group (JUDD et al., 1999).

Tabela 2 - Informações sobre a intensidade amostral nos fragmentos.

Table 2 - Information on the sampling intensity in the fragments

\begin{tabular}{|c|c|c|c|c|}
\hline Fragmento & $\begin{array}{l}\text { Área do } \\
\text { fragmento } \\
\text { (ha) }\end{array}$ & $\begin{array}{c}\text { Número de } \\
\text { parcelas }\end{array}$ & $\begin{array}{c}\text { Área amos- } \\
\text { tral (ha) }\end{array}$ & $\begin{array}{l}\text { Área amos- } \\
\text { tral (\%) }\end{array}$ \\
\hline J10 & 4922,10 & 60 & 6,0 & 0,12 \\
\hline $\mathrm{J} 15$ & 1557,41 & 52 & 5,2 & 0,33 \\
\hline $\mathrm{J} 14$ & 931,93 & 39 & 3,9 & 0,42 \\
\hline $\mathrm{J} 108$ & 824,15 & 50 & 5,0 & 0,61 \\
\hline P115 & 560,54 & 10 & 1,0 & 0,18 \\
\hline $\mathrm{J} 13$ & 488,26 & 36 & 3,6 & 0,74 \\
\hline J109 & 408,97 & 20 & 2,0 & 0,49 \\
\hline $\mathrm{J} 20$ & 406,89 & 15 & 1,5 & 0,37 \\
\hline P95 & 364,73 & 20 & 2,0 & 0,55 \\
\hline M134 & 311,05 & 10 & 1,0 & 0,32 \\
\hline $\mathrm{J} 135$ & 308,53 & 10 & 1,0 & 0,32 \\
\hline J96 & 307,30 & 22 & 2,2 & 0,72 \\
\hline J86 & 284,55 & 30 & 3,0 & 1,05 \\
\hline $\mathrm{J} 110$ & 209,73 & 16 & 1,6 & 0,76 \\
\hline P94 & 201,07 & 14 & 1,4 & 0,70 \\
\hline $\mathrm{J} 21$ & 191,41 & 15 & 1,5 & 0,78 \\
\hline J139 & 180,19 & 15 & 1,5 & 0,83 \\
\hline P119 & 156,57 & 15 & 1,5 & 0,96 \\
\hline $\mathrm{J} 16$ & 143,98 & 20 & 2,0 & 1,39 \\
\hline S111 & 141,89 & 10 & 1,0 & 0,70 \\
\hline $\mathrm{J} 114$ & 118,02 & 13 & 1,3 & 1,10 \\
\hline $\mathrm{J} 120$ & 114,07 & 10 & 1,0 & 0,88 \\
\hline $\mathrm{J} 12$ & 111,90 & 21 & 2,1 & 1,88 \\
\hline $\mathrm{J} 11$ & 87,67 & 25 & 2,5 & 2,85 \\
\hline M112 & 78,86 & 10 & 1,0 & 1,27 \\
\hline M140 & 20,59 & 10 & 1,0 & 4,86 \\
\hline Total & 13432,39 & 568,00 & 56,80 & 0,42 \\
\hline
\end{tabular}

\subsection{Similaridade florística}

A vegetação foi estratificada em relação ao padrão florístico predominante, agrupando-se os fragmentos por meio da similaridade de espécies existente entre eles. Calculou-se o coeficiente de Sorensen a partir de uma matriz binária com dados florísticos de cada fragmento. Segundo Kent e Coker (1992), esse quociente é preferido por fornecer pesos para as espécies mais comuns.

Após definir a medida de similaridade a ser utilizada, a etapa seguinte foi usar uma técnica de agrupamento para formação dos grupos. Para isso, utilizou-se o algoritmo de médias ponderadas (UPGMA - Unweighted PairGroups Method Average) como medida de ligação entre os fragmentos. $\mathrm{O}$ resultado final desse procedimento foi expresso por meio de um gráfico tipo dendrograma, e os grupos de fragmentos formados foram estipulados a partir de um corte de $25 \%$ no nível de informação no eixo x do dendrograma. Segundo Mueller-Dombois e Ellenberg (1974) nesse nível de corte já ocorre a fusão de grupos semelhantes. A análise de agrupamento foi realizada por meio do programa PC-ORD versão 4.14 (MCCUNE; MEFFORD, 1999).

\subsection{Diversidade e equabilidade}

Calcularam-se os índices de diversidade de Shannon-Wiener (LUDWIG e REYNOLDS, 1988) e de equabilidade de Pielou (POOLE, 1974) para analisar a variação das espécies dentro da área estudada, com vistas a uma melhor compreensão da distribuição das espécies presentes nas Bacias. Para Odum (1988) a diversidade de grupos está associada a uma relação entre o número de grupos (riqueza) e a distribuição do número de indivíduos entre os grupos (equabilidade). Esta definição está explicitada nos índices de Shannon e de Pielou, que conjugam estas duas variáveis.

\section{RESULTADOS E DISCUSSÃO}

\subsection{Análise da similaridade florística}

A Figura 1 mostra os resultados do agrupamento obtido com base no coeficiente de Sorensen, o qual proporcionou a formação de seis grupos floristicamente semelhantes (Tabela 3). O grupo florístico $1 \mathrm{com} 83$ espécies foi formado pelos três fragmentos de Floresta Estacional Decidual, sendo que dezoito delas foram comuns

Cerne, Lavras, v. 20, n. 1, p. 1-10, jan./mar. 2014 


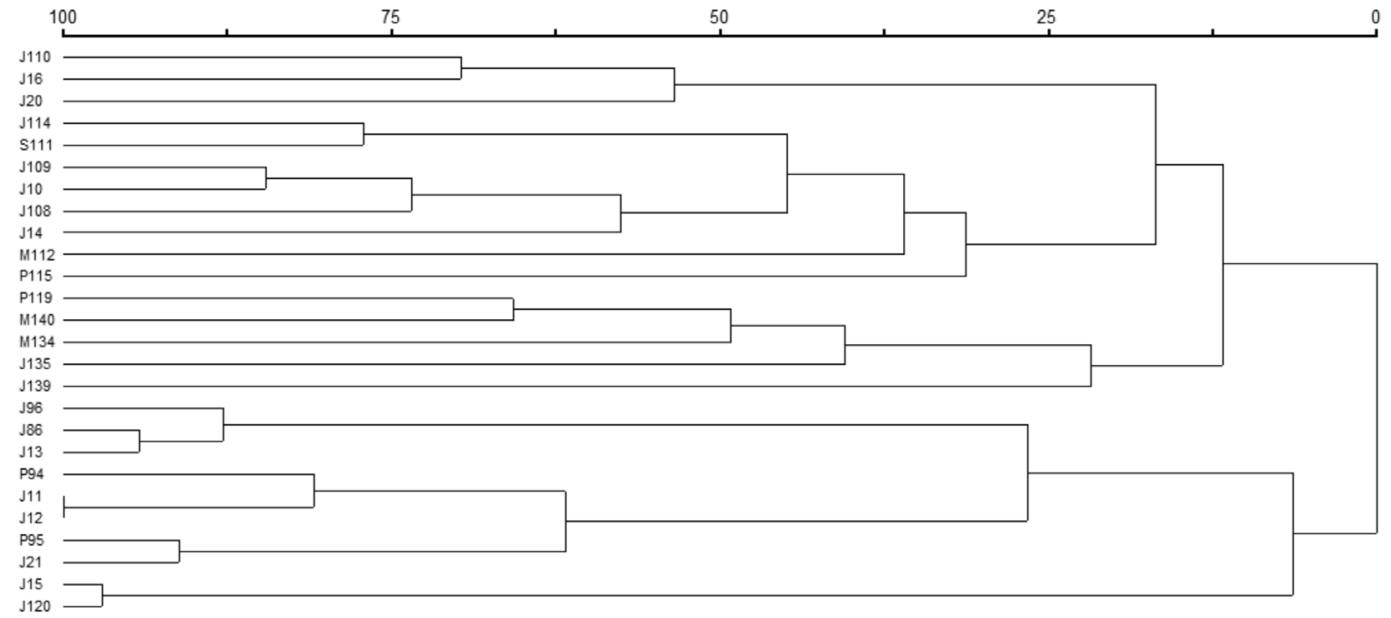

Figura 1 - Dendrograma de Similaridade florística obtido para as espécies presentes nos 26 Fragmentos, utilizando o método de medias ponderado por grupo (UPGMA).

Figure 1 - Dendrogram of floristic similarity obtained for the species present in 26 fragments, using the method of averages weighted by group (UPGMA).

na associação de pelo menos dois fragmentos. Entretanto, apenas três espécies ligaram todos os fragmentos desse grupo: Deguelia costata (Benth.) Az.-Tozzi, Machaerium acutifolium Vogel e Myracrodruon urundeuva Allemão. As espécies Tabebuia roseoalba (Ridl.) Sandwith, Combretum leprosum Mart., Eugenia uniflora L., Capparis yco Mart. e Chloroleucon tortum (Mart.) Pittier, também merecem destaque, pois não ocorreram em nenhum dos demais fragmentos estudados. Para Leitão-Filho (1982) os elementos exclusivos caracterizam determinados tipos florestais e os elementos de interpenetração auxiliam no estabelecimento dos limites de cada formação. Dessa forma, a análise desses dois componentes é relevante, auxiliando na discriminação dos grupos.

Entre os fragmentos do grupo florístico 2 ocorreram 230 espécies comuns entre si, considerando a combinação de, no mínimo, dois fragmentos, sendo que apenas a espécie Roupala montana Aubl. é comum a todos eles. De acordo com o dendrograma, os fragmentos J114 e S111 demonstraram alta similaridade, pois só poderão ser dissociados com um corte depois de $75 \%$ no nível de informação, sendo que entre esses dois fragmentos ocorreram 34 espécies comuns com destaque para Trichilia pallida Sw., Nectandra oppositifolia Nees, Miconia cinnamomifolia (DC.) Naudin e Croton celtidifolius Baill. que ocorreram unicamente nesses fragmentos.

Outro sub-grupo coeso foi formado pelos fragmentos J10, J108 e J109 que também requerem um corte rigoroso no eixo x para serem separados. Entre esses fragmentos houve 36 espécies em comum, destacando-se Myrcia mutabilis (O.Berg) N.Silveira, Licania kunthiana Hook.f., Cupania emarginata Cambess., Ilex theezans Mart. ex Reissek, Micropholis venulosa (Mart. \& Eichler) Pierre, Pseudopiptadenia warmingii (Benth.) G.P.Lewis \& M.P.Lima, Sapium glandulosum (L.) Morong, Ternstroemia brasiliensis Cambess., Schefflera calva (Cham.) Frodin \& Fiaschi, Simarouba amara Aubl. e Jacaranda cuspidifolia Mart. ex A.DC., que foram exclusivas dos mesmos. Esse resultado mostra que o fragmento J108, apesar de estar inserido dentro das Florestas Estacionais Semideciduais, sofre pressão do Cerrado sensu stricto dado a grande semelhança florística apresentada.

No grupo florístico 3 ocorreram 60 espécies comuns na combinação de pelo menos dois fragmentos. As espécies que associaram os 4 fragmentos foram Astronium fraxinifolium Schott ex Spreng. e Melanoxylon brauna Schott, sendo que essa última teve maior impacto na ligação dos fragmentos, uma vez que sua única ocorrência fora das Florestas Estacionais Semideciduais foi no fragmento P119.

O grupo florístico 4 é composto apenas do fragmento J139 (Floresta Estacional Semidecidual), demonstrando que ele possui composição florística distinta dos demais fragmentos. Ocorreram 24 espécies exclusivas, o que representa $16,90 \%$ do total de espécies desse fragmento.

Entre os fragmentos do grupo 5 ocorreram 72 espécies comuns, sendo que a associação de todos os fragmentos do grupo 5 se deu por meio de 12 espécies:

Cerne, Lavras, v. 20, n. 1, p. 1-10, jan./mar. 2014 
Acosmium dasycarpum (Vogel) Yakovlev, Byrsonima coccolobifolia Kunth, Caryocar brasiliense Cambess., Dalbergia miscolobium Benth., Enterolobium gummiferum (Mart.) J.F.Macbr., Eugenia dysenterica DC., Hymenaea stignocarpa Mart. ex Hayne, Lafoensia pacari A.St.-Hil., Machaerium opacum Vogel, Plathymenia reticulata Benth., Qualea grandiflora Mart.,e Stryphnodendron adstringens (Mart.) Cov. que foram de ampla distribuição em toda área estudada. Nesse grupo não ocorreu nenhuma espécie exclusiva, evidenciando a prioridade das espécies comuns na formação dos grupos pelo coeficiente de Sorensen.

Tabela 3 - Grupos de fragmentos formados com base no coeficiente de Sorensen.

Table 3 - Groups of fragments formed based on the coefficient of

Sorensen.

\begin{tabular}{|c|c|c|c|}
\hline Grupos & Fragmentos & Município & Fisionomia predominante \\
\hline \multirow{3}{*}{1} & J110 & Araçuaí & Floresta Estacional Decidual \\
\hline & $\mathrm{J} 16$ & Salinas & Floresta Estacional Decidual \\
\hline & $\mathrm{J} 20$ & Jenipapo de Minas & Floresta Estacional Decidual \\
\hline \multirow{8}{*}{2} & J114 & Aricanduva & $\begin{array}{l}\text { Floresta Estacional Semide- } \\
\text { cidual }\end{array}$ \\
\hline & S111 & Frei Gaspar & $\begin{array}{l}\text { Floresta Estacional Semide- } \\
\text { cidual }\end{array}$ \\
\hline & J109 & Jequitinhonha & Cerrado sensu stricto \\
\hline & $\mathrm{J} 10$ & Leme do Prado & Cerrado sensu stricto \\
\hline & J108 & Jequitinhonha & $\begin{array}{l}\text { Floresta Estacional Semide- } \\
\text { cidual }\end{array}$ \\
\hline & J14 & Minas Novas & Cerrado sensu stricto \\
\hline & M112 & Teófilo Otoni & $\begin{array}{l}\text { Floresta Estacional Semide- } \\
\text { cidual }\end{array}$ \\
\hline & P115 & Ninheira & $\begin{array}{c}\text { Floresta Estacional Semide- } \\
\text { cidual }\end{array}$ \\
\hline \multirow{4}{*}{3} & P119 & Indaiabira & Cerrado sensu stricto \\
\hline & M140 & Águas Formosas & $\begin{array}{l}\text { Floresta Estacional Semide- } \\
\text { cidual }\end{array}$ \\
\hline & M134 & Nanuque & $\begin{array}{l}\text { Floresta Estacional Semide- } \\
\text { cidual }\end{array}$ \\
\hline & J135 & Jacinto & $\begin{array}{c}\text { Floresta Estacional Semide- } \\
\text { cidual }\end{array}$ \\
\hline 4 & J139 & $\begin{array}{l}\text { São Gonçalo do Rio } \\
\text { Preto } \\
\end{array}$ & $\begin{array}{c}\text { Floresta Estacional Semide- } \\
\text { cidual }\end{array}$ \\
\hline \multirow{8}{*}{5} & J96 & Itacambira & Cerrado sensu stricto \\
\hline & $\mathrm{J} 86$ & Fruta de Leite & Campo cerrado \\
\hline & $\mathrm{J} 13$ & Itamarandiba & Cerrado sensu stricto \\
\hline & P94 & Montezuma & Cerrado sensu stricto \\
\hline & $\mathrm{J} 11$ & Olhos d'água & Cerrado sensu stricto \\
\hline & $\mathrm{J} 12$ & Olhos d'água & Cerrado sensu stricto \\
\hline & P95 & Rio Pardo de Minas & Campo cerrado \\
\hline & $\mathrm{J} 21$ & Salinas & Cerrado sensu stricto \\
\hline \multirow[b]{2}{*}{6} & $\mathrm{~J} 15$ & Turmalina & Campo cerrado \\
\hline & $\mathrm{J} 120$ & Coronel Murta & Cerrado sensu stricto \\
\hline
\end{tabular}

O grupo florístico 6 foi formado pelos fragmentos J15 (Campo cerrado) e J120 (Cerrado sensu stricto). Entre esses fragmentos ocorreram 26 espécies comuns, sendo que 15 delas são de ampla distribuição nas Bacias. Esse grupo foi o mais coeso, pois a sua dissolução só ocorrerá com um corte perto de $100 \%$ no eixo x do dendrograma.

Esses resultados indicam que os laços florísticos que unem os fragmentos são determinados de forma mais contundente pela composição florística do que pela classificação quanto à fisionomia predominante. Isto mostra que a sobreposição e translocação de elementos permitem a formação de vegetações miscigenadas, apesar da fisionomia aparentemente uniforme.

A distribuição geográfica dos grupos pode ser visualizada na Figura 2. De uma forma geral, os grupos estão distribuídos no sentido longitudinal do mapa, sendo que no eixo central ocorre a dispersão de grupos de diferentes tipos fisionômicos. Essa distribuição sugere que a região compreendida entre as longitudes $42^{\circ} 49^{\prime} 09^{\prime \prime}$ (J15) e $42^{\circ} 07^{\prime} 45^{\prime \prime}$ (J21), possui características geo-climáticas que favorecem a distribuição de espécies de diversas formações vegetais. Esse comportamento caracteriza regiões de transição, onde a pressão que as fisionomias exercem entre si influencia no padrão florístico dessas áreas.

Além da influência das formações vegetais adjacentes, a composição florística, de forma geral, pode ser influenciada por vários fatores em diferentes escalas. Segundo Ivanauskas (2002), as formações florestais refletem as condições ambientais do meio, permitindo a existência permanente de uma comunidade, e com a qual, cada população interage. Dessa forma, os limites entre os grupos formados talvez não seja apenas resultado dos aspectos fisionômicos, florísticos e estruturais, mas também dos fatores abióticos determinantes de cada uma das formações florestais.

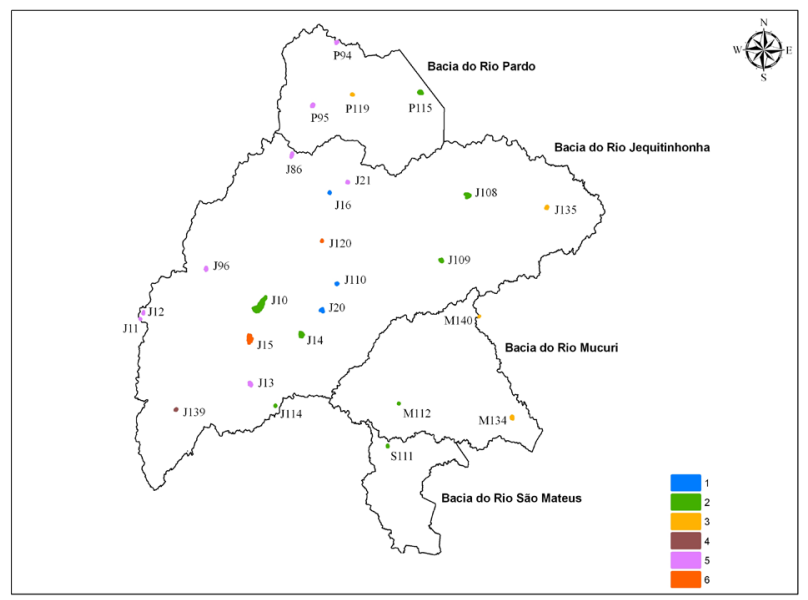

Figura 2 - Localização geográfica dos grupos de fragmentos de acordo com o coeficiente de similaridade de Sorensen.

Figure 2 - Geographical location of groups of fragments according to the Sorensen similarity coefficient.

Cerne, Lavras, v. 20, n. 1, p. 1-10, jan./mar. 2014 


\subsection{Análise da diversidade e equabilidade}

\subsubsection{Na área estudada}

A amplitude do índice de diversidade de Shannon nas Bacias foi de 2,236 (J16, Floresta Estacional Decidual) a 4,523 (J114, Floresta Estacional Semidecidual) e a variação do índice de equabilidade de Pielou foi de 0,616 (J16) a 0,850 (J114) (Tabela 4). De modo geral, as Florestas Estacionais Semideciduais apresentaram os maiores valores, obtendo-se uma média de 3,373 (H') e 0,770 (J').

Tabela 4 - Comportamento dos índices de Diversidade e Equabilidade, nos fragmentos estudados.

Table 4 - Behavior shown by indicators of Diversity and equability, in the studied fragments.

\begin{tabular}{ccccc}
\hline Fragmento & $\mathrm{N}$ & $\mathrm{n}$ & $\mathrm{H}{ }^{\prime}$ & $\mathrm{J}$ \\
\hline J114 & 2308 & 204 & 4,523 & 0,850 \\
J108 & 9104 & 221 & 4,130 & 0,765 \\
J139 & 1979 & 142 & 4,003 & 0,808 \\
M112 & 1051 & 98 & 3,791 & 0,829 \\
J15 & 2591 & 103 & 3,564 & 0,769 \\
J10 & 16276 & 140 & 3,537 & 0,717 \\
J12 & 3403 & 70 & 3,371 & 0,793 \\
J109 & 2830 & 104 & 3,320 & 0,715 \\
J11 & 3709 & 72 & 3,281 & 0,767 \\
J96 & 1420 & 57 & 3,206 & 0,793 \\
J135 & 621 & 46 & 3,193 & 0,834 \\
S111 & 882 & 66 & 3,158 & 0,754 \\
P119 & 2251 & 86 & 3,128 & 0,702 \\
J13 & 3480 & 55 & 3,023 & 0,754 \\
J120 & 3657 & 47 & 2,950 & 0,766 \\
P94 & 1227 & 45 & 2,861 & 0,752 \\
J86 & 1385 & 33 & 2,759 & 0,789 \\
P115 & 1746 & 45 & 2,707 & 0,711 \\
J14 & 4078 & 56 & 2,647 & 0,657 \\
M134 & 1051 & 68 & 2,644 & 0,627 \\
M140 & 757 & 32 & 2,630 & 0,759 \\
P95 & 405 & 28 & 2,612 & 0,784 \\
J110 & 1264 & 32 & 2,541 & 0,733 \\
J20 & 1384 & 34 & 2,502 & 0,709 \\
J21 & 1404 & 37 & 2,478 & 0,686 \\
J16 & 3163 & 2,236 & 0,619 \\
\hline & & & & \\
\hline
\end{tabular}

Onde: $\mathrm{N}$ - número de indivíduos, $\mathrm{n}$ - número de espécies, $\mathrm{H}^{\prime}$ índice de Shannon e J'- Equabilidade de Pielou
Segundo Dias (2005), altos valores dos índices de diversidade e equabilidade indicam comunidades mais uniformes, onde a dominância de um ou poucos grupos é mais atenuada. Por outro lado, os valores de diversidade encontrados nos fragmentos P115, M134 e M140 não corresponderam aos resultados obtidos por outros autores para as Florestas Estacionais Semideciduais (LOPES et al., 2002; SILVA et al., 2004; MACHADO et al., 2004; IMANA-ENCINAS et al., 2007) onde os índices de Shannon encontrados foram superiores a 3,500 , o que indica que esses fragmentos podem ter sofrido algum tipo de intervenção antrópica. Segundo Carvalho e MarquesAlves (2008), distúrbios freqüentes são representativos e alteram a vegetação, sua distribuição de espécies, abundância e estádio sucessional.

Já os fragmentos de Floresta Estacional Decidual (J16, J20 e J110) apresentaram os menores valores de diversidade, juntamente com um fragmento de Cerrado sensu stricto (J21), localizado no mesmo município do fragmento J16. A média de valores encontrados foi 2,426 (H') e 0,687 (J'). Esses resultados permitem inferir que poucas comunidades predominam no padrão florístico dessas populações. Segundo Gentry (1995) as Florestas Estacionais Deciduais são menos diversas, podendo apresentar menos da metade das espécies arbóreas encontradas em outras florestas tropicais. Mas, realizando-se comparações com outros estudos, comprova-se que os fragmentos estudados apresentaram baixa diversidade florística. Por exemplo, Gomide (2004) estudou a diversidade e equabilidade de fragmentos de Florestas Estacionais Deciduais situados na Bacia do rio São Francisco e encontrou valores médios de 3,030, para o índice de Shannon e 0,785, para a equabilidade de Pielou. Santos et al. (2007) estudaram a florística e a estrutura de um remanescente de Floresta Estacional Decidual localizado no Parque Municipal da Sapucaia no município de Montes Claros e encontrou valores de 3,3 para H'e de 0,8 para J'.

Os fragmentos de Cerrado sensu stricto apresentaram uma média de 3,072 (H’) e 0,736 (J). O valor do índice de diversidade de Shannon $\left(\mathrm{H}^{\prime}\right)$ foi similar ao encontrado em outros levantamentos em áreas de Cerrado sensu stricto (SAPORETTI Jr. et al., 2003; FIDELIS; GODOY, 2003; ASSUNÇÃO; FELFILI, 2004), que em geral apresentam o H' em torno de 3,00. O fragmento com maior diversidade foi o $\mathrm{J} 10$, contudo a equabilidade foi inferior à média encontrada nessa fisionomia, podendo-se inferir que

Cerne, Lavras, v. 20, n. 1, p. 1-10, jan./mar. 2014 
poucas comunidades predominam no padrão florístico desse fragmento.

Nos fragmentos de Campo cerrado os valores médios foram de 2,978 e 0,791 para os índices de Shannon e Pielou, respectivamente. Os resultados encontrados para os parâmetros de diversidade só foram maiores que os valores obtidos para as Florestas Estacionais Deciduais, entretanto o índice de equabilidade foi o maior entre todas as fisionomias mencionadas. O fragmento J15 se destacou situando-se em quinto lugar na Tabela 3, elevando o valor da média da diversidade. Contudo, em relação à equabilidade, os fragmentos dessa fisionomia apresentaram valores acima de 0,76 demonstrando maior equilíbrio entre o número de espécies e indivíduos, o que reduz a dominância ecológica entre as populações. Esse fato pode significar um maior grau de conservação desses remanescentes em comparação às demais fisionomias avaliadas nesse estudo.

\subsubsection{No grupo florístico 1}

Os índices de diversidade de Shannon para os fragmentos variaram de 2,236 (J16) a 2,541 (J110). O fragmento com a menor diversidade foi J16, com 0,184. A equabilidade de Pielou apresentou os seguintes valores: 0,619 (J16); 0,709 (J20) e 0,733 (J110). Os valores encontrados foram os menores encontrados para toda área estudada demonstrando que as Florestas Estacionais Deciduais possuem índices de diversidade menores em relação às demais fitofisionomias analisadas. $\mathrm{O}$ fragmento J16 do município de Salinas apresentou o menor índice de Equabilidade, indicando uma desproporção entre as espécies quanto ao número de indivíduos. Isso sugere que existe uma predominância de algumas espécies, o que propicia um desequilíbrio nessa vegetação.

As espécies com um grande número de indivíduos e que provocaram um baixo valor deste parâmetro no fragmento J16 foram: Combretum leprosum Mart. (1166) e Myracrodruon urundeuva Allemão (588) que representaram 55,46\% do número de indivíduos. Essas espécies promoveram a redução da diversidade e o aumento do número de espécies raras, o que pode ser exemplificado por 7 espécies que tiveram apenas um representante.

\subsubsection{No grupo florístico 2}

Os índices de Shannon variaram de 2,647 (J14) a 4,523 (J114) e a equabilidade de Pielou apresentou valores no intervalo de $0,657(\mathrm{~J} 14)$ até $0,850(\mathrm{~J} 114)$, indicando haver disparidade de comportamento dentro desse grupo, demonstrando que a estrutura das populações é variável entre os fragmentos. $\mathrm{O}$ fragmento de maior diversidade e equabilidade foi o J114, onde ocorreram 2.308 indivíduos distribuídos em 204 espécies. Em contrapartida, no fragmento $\mathrm{J} 14$ foram amostrados 4.078 indivíduos que estão distribuídos em 56 espécies, sendo que as espécies Rourea induta Planch. (979), Ormosia arborea (Vell.) Harms (402), Jacaranda macrantha Cham. (390) e Machaerium brasiliense Vogel (333) representam 51,6\% do total de indivíduos.

\subsubsection{No grupo florístico 3}

Os valores de Shannon variaram de 2,630 (M140) a 3,193 (J135) e o fragmento J135 apresentou o maior valor $(0,834)$ para o índice de equabilidade. Nesse fragmento, foram amostrados 621 indivíduos em 46 espécies, o que propiciou uma elevada equabilidade.

Por outro lado, o fragmento M134 alcançou o menor valor de equabilidade $(0,627)$ e um dos menores valores de diversidade $(0,644)$. Esses resultados foram próximos aos das Florestas Estacionais Deciduais, indicando que o comportamento das espécies mostra dominância ecológica entre as populações do fragmento. A espécie Astronium fraxinifolium Schott ex Spreng. com 422 indivíduos predominou nesse fragmento, representando $40,16 \%$ de um total de 1.051 indivíduos. Já o fragmento M140 apresentou a menor diversidade de acordo com Shannon $(2,630)$, sendo que nesse fragmento ocorreu o menor número de espécie do grupo (32).

\subsubsection{No grupo florístico 4}

O fragmento J139 apresentou valores elevados de diversidade e equabilidade, comparando-se com os demais fragmentos estudados. O índice de Shannon $(4,003)$ foi o terceiro maior de acordo com a Tabela 3. Já o índice de equabilidade foi o quarto maior com 0,808. Nesse fragmento ocorreram 1.979 indivíduos, distribuídos em 142 espécies.

Reis et al. (2007) estudaram a diversidade de quatro fragmentos de Floresta Estacional Semidecidual situados na zona da mata mineira e encontraram valores para o índice de Shannon que variaram de 3,91 a 4,7. Considerando esses valores, pode-se inferir que o fragmento J139 apresentou uma diversidade compatível com a fisionomia.

Cerne, Lavras, v. 20, n. 1, p. 1-10, jan./mar. 2014 


\subsubsection{No grupo florístico 5}

Neste grupo florístico os valores de diversidade de Shannon variaram de 2,478 (J21) a 3,371(J12) e o índice de equabilidade de Pielou situou-se na faixa de 0,686 (J21) a 0,793 (J96). De acordo com esses resultados o fragmento J21 apresentou a menor diversidade e equabilidade do grupo. Nesse fragmento ocorreram 37 espécies, sendo que Dalbergia miscolobium Benth., Plathymenia reticulata Benth. e Bowdichia virgilioides Kunth somaram 756 indivíduos, o que representa $53,85 \%$ do total.

Já os fragmentos J12 e J96 tiveram os maiores valores de diversidade e ambos apresentaram o mesmo valor de equabilidade $(0,793)$. De acordo com o índice de Shannon o fragmento com maior diversidade foi o J12, onde ocorreram 70 espécies. Já em relação ao índice de Simpson o fragmento J96 possui a maior diversidade do grupo com 57 espécies.

\subsubsection{No grupo florístico 6}

Nos fragmentos J120 e J15 os valores do índice de Shannon foram de 2,950 e 3,564, respectivamente. Já em relação à equabilidade, os valores variaram de 0,799 (J120) a 0,769 (J15). A amplitude alcançada para os índices de diversidade foi elevada, enquanto que para a equabilidade essa variação foi de apenas 0,0003 . No fragmento J120 ocorreram 3.657 indivíduos em 47 espécies, sendo que a espécie Machaerium dimorphandrum Hoehne apresentou 1.587 indivíduos o que representou 43,40\% dos indivíduos desse fragmento. Esses resultados contribuíram para a baixa equabilidade encontrada. Já o fragmento J15 apresentou 103 espécies representadas por 2.591 indivíduos.

\section{REFERÊNCIAS}

ASSUNÇÃO, S. L.; FELFILI, J. M. Fitossociologia de um fragmento de cerrado sensu stricto na APA do Paranoá, DF, Brasil. Acta Botânica Brasilica, Feira de Santana, v.18, p.903-909, 2004.

BIERREGAARD, R. O. Jr; DALE, V. H. Islands in a ever-changing sea: the ecological and socioeconomic dynamics of Amazonian rainforest IN: SCHELLAS, J.; GREENBERG, R (Ed.). Forest patches in tropical landscapes. Washington: Island Press,p. 1996. p. 187-204.

Cerne, Lavras, v. 20, n. 1, p. 1-10, jan./mar. 2014
CARVALHO, D.A.; OLIVEIRA-FILHO, A.T.; VILELA, E.A., CURI, N.; VAN DEN BERG, E.; FONTES, M.A.L.; BOTEZELLI, L. Distribuição de espécies arbóreo-arbustivas ao longo de um gradiente de solos e topografia em um trecho de floresta ripária do Rio São Francisco em Três Marias, MG, Brasil. Revista Brasileira de Botânica, São Paulo, v.28, n.2, p.329-345, abr.-jun. 2005.

CARVALHO, J. C. Gestão florestal em Minas Gerais. In: LOPES, I. V.; BASTOS FILHO, G. S.; BILLEER, D.; BALE, M. (Orgs.). Gestão ambiental no Brasil: experiência e sucesso. Rio de Janeiro: Fundação Getúlio Vargas, 1996.

CARVALHO, L. M. T.; SCOLFORO, J. R. S.. Zoneamento Ecológico Econômico do Estado de Minas Gerais. Lavras: Editora UFLA, 2007 (Atlas digital).

CARVALHO, A. R.; MARQUES-ALVES, S.

Diversidade e índice sucessional de uma vegetação de Cerrado sensu stricto na universidade estadual de GoiásUEG, campus de Anápolis. Revista Árvore, Viçosa, v.32, n.1, p.81-90, 2008.

COSTA, C. R. M.; HERRMANN, G.; MARTINS, C. S.; LINS, L. V.; LAMAS, I.R. Biodiversidade em Minas Gerais: um atlas para sua conservação. Belo Horizonte: Fundação Biodiversitas, 1998.

DIAS, A.C. Composição florística, fitossociologia, diversidade de espécies arbóreas e comparação de métodos de amostragem na floresta ombrófila densa do parque estadual Carlos Botelho, SP/Brasil. 2005. 184f. Tese (Doutorado em recursos florestais) - Escola Superior de Agricultura "Luiz de Queiroz", Universidade de São Paulo, Piracicaba.

FIDELIS, A. T.; GODOY, S. A. P. Estrutura de um cerrado strico sensu na Gleba Cerrado Pé-de-Gigante, Santa Rita do Passa Quatro, SP. Acta Botanica Brasilica, Feira de Santana, v.17, n.4, p.531-539, 2003.

GENTRY, A. H.. Patterns of diversity and floristic composition in neotropical montane forests. In: CHURCHILL, S. P.; BALSLEY, H.; FORERO, E.; LUTEYN, J. L. (Eds.). Biodiversity and Conservation 
of Neotropical Montane Forests: Proceedings of Neotropical Montane Forest Biodiversity and Conservation Symposium. New York: The New York Botanical Garden, 1995. p. 103-126

GOMIDE, L. R.. Um modelo fitogeografico para a bacia do rio São Francisco, em Minas Gerais. 2004. 244f. Dissertação (Mestrado em Engenharia Florestal) Universidade Federal de Lavras, Lavras.

IMAÑA-ENCINAS, J.; MACEDO, L. A.; PAULA, J. E. Florística e fitossociologia de um trecho da floresta estacional semidecidual na área do ecomuseu do cerrado em Pirenópolis, Goiás. Cerne, Lavras, v. 13, n. 3, p. 308-320, jul./set. 2007.

IVANAUSKAS, N. M. Caracterização da vegetação na área de contato entre formações florestais em Gaúcha do Norte-MT. 2002. 185f. Tese (Doutorado em Biologia Vegetal) - Universidade Estadual de Campinas, Campinas.

JUDD, W. S.; CAMPBELL, C. S.; KELLOG, E. A.; STEAVENS, P. F. Plant systematics: a phylogenetic approach. Massachusetts: Sinauer Associates, 1999. $464 p$.

KAGEYAMA, P.; GANDARA, F. B. Revegetação de áreas ciliares. In: RODRIGUES, R. R.; LEITÃO FILHO, H. F. (Eds.). Matas Ciliares: conservação e recuperação. São Paulo: Editora da Universidade de São Paulo/Fapesp, 2000. p.249-269.

KENT, M.; COKER, P. Vegetation description and analysis: a practical approach. Chichester: J. Wiley, 1992. $363 \mathrm{p}$.

LOPES, P. W.; SILVA, A. F.; SOUZA, A. L.; MEIRANETO, J. A. A. Estrutura fitossociológica de um trecho de vegetação arbórea no Parque Estadual do Rio Doce - Minas Gerais, Brasil. Acta Botânica Brasílica, Porto Alegre, v. 16, n. 4, p. 443-456, 2002.

LUDWIG, J. A.; REYNOLDS, J. F. Statistical ecology: a primer on methods and computing. New York: J. Wiley, 1988. 337p.

MACHADO, E. L. M.; OLIVEIRA FILHO, A. T.; CARVALHO, W. A.; SOUZA, J. S.; BORÉM, R. A. T.; BORTEZELLI, L. Análise comparativa da estrutura e flora do compartimento arbóreo-arbustivo de um remanescente florestal na fazenda Beira Lago,
Lavras, MG. Revista Árvore, Viçosa, v. 28, n. 4, p. 499$516,2004$.

MCCUNE, B.; MEFFORD, M. J. PC-ORD.

Multivariate analysis of ecological data, version 4. MjM Software Design, Gleneden Beach, OR, US. 1999

MUELLER-DOMBOIS, D.; ELLENBERG, H. Aims and methods of vegetation ecology. New York: Willey and Sons, 1974. 574 p.

ODUM, E. P. Ecologia. Rio de Janeiro: Guanabara Koogan, 1988. 434 p.

POOLE, R. W. An introduction to quantitative ecology. Tokyo: McGraw-Hill Kogakusha, 1974. 532p.

REIS, H.; SCOLFORO, J. R. S.; OLIVEIRA, A. D.; OLIVEIRA FILHO, A. T.; MELLO, J. M. Análise da composição florística, diversidade e similaridade de fragmentos de mata atlântica em Minas Gerais. Cerne, Lavras, v. 13, n. 3, p. 280-290, jul./set. 2007.

SANTOS, R. M.; VIEIRA, F. A.; GUSMÃO, E.; NUNES, Y. R. F. Florística e estrutura de uma floresta estacional decidual, no Parque Municipal da Sapucaia, Montes Claros (MG). Cerne, Lavras, v. 13, n. 3, p. 248256, jul./set. 2007.

SAPORETTI JR, A.; MEIRA NETO, J. A.; ALMADO, R. de P. Fitossociologia de cerrado sensu strito no município de Abaeté, MG. Revista Árvore, Viçosa, MG, v. 27, n. 3, p. 413-419, 2003.

SCOLFORO, J. R. S.; CARVALHO, L. M. T. Mapeamento e inventário da flora nativa e dos reflorestamentos de Minas Gerais. Lavras: UFLA, 2006. 288p.

SILVA, J. M. C.; TABARELLI, M. Tree species impoverishment and the future flora of the Atlantic forest of northeast Brazil. Nature, v. 404, p.72-74, 2000.

SILVA, N. R. S.; MARTINS, S. V.; MEIRA NETO, J. A. A. Composição florística e estrutura de uma floresta estacional semidecidual montana em Viçosa, MG.

Revista Árvore, Viçosa, v. 28, n. 3, p. 397-405, 2004.

Recebido: 13 de julho de 2011; aceito: 11 de abril de 2013.

Cerne, Lavras, v. 20, n. 1, p. 1-10, jan./mar. 2014 
